\title{
Listagem de pareceristas ad hoc do volume 13 (ano 2015)
}

Ana Cristina Borges de Oliveira / Universidade Federal de Minas Gerais

Ada Ávila Assunção / Universidade Federal de Minas Gerais

Adriana Kelly dos Santos / Fundação Oswaldo Cruz

Adriano Beiras / Universidade Federal de Santa Catarina

Alan Vendrame / Universidade Federal de São Paulo

Alberto Durán González / Universidade Estadual de Londrina

Aline Guerra Aquilante / Universidade Federal de São Carlos

Alva Helena de Almeida / Secretaria Municipal da Saúde de São Paulo

Ana Lúcia de Assis Simões / Universidade Federal do Triângulo Mineiro

Ana Lucia de Moura Pontes / Fundação Oswaldo Cruz

Ana Margarida Barreto Campello / Fundação Oswaldo Cruz

Antonio Germane Alves Pinto / Universidade Regional do Cariri

Arlinda Barbosa Moreno / Fundação Oswaldo Cruz

Bertha Cruz Enders / Universidade Federal do Rio Grande do Norte

Bianca Ramos Marins / Fundação Oswaldo Cruz

Carla Ribeiro Guedes / Universidade Federal Fluminense

Carlito Lopes Nascimento Sobrinho / Universidade Estadual de Feira de Santana

Carlos Dimas Martins Ribeiro / Universidade Federal Fluminense

Cassius Schnell Palhano Silva / Fundação Oswaldo Cruz

Cecy Dunshee de Abranches / Fundação Oswaldo Cruz 
Claudia Maria Filgueiras Penido / Universidade Federal de Minas Gerais

Cristina Pereira Gaglianone / Universidade Federal de São Paulo

Daniela Dellagrave / Universidade Federal do Rio Grande do Sul

Deborah Uhr / Secretaria Municipal de Saúde do Rio de Janeiro

Deise Mancebo / Universidade do Estado do Rio de Janeiro

Denise Rangel Ganzo de Castro Aerts / Universidade Luterana do Brasil

Elaine Silva Miranda / Fundação Oswaldo Cruz

Eliane Marina Palhares Guimarães / Universidade Federal de Minas Gerais

Eliane Matos / Universidade Federal de Santa Catarina

Eliane Vianna / Universidade Federal do Rio de Janeiro

Elisabete de Fátima Polo de Almeida Nunes / Universidade Estadual de Londrina

Elma Lourdes Campos Pavone Zoboli / Universidade de São Paulo

Estela Marcia Saraiva Campos / Universidade Federal de Juiz de Fora

Etelcia Moraes Molinaro / Fundação Oswaldo Cruz

Fábio Bruno de Carvalho / Pontifícia Universidade Católica de Campinas

Fabíola Zioni / Universidade de São Paulo

Fernanda de Oliveira Sarreta / Universidade Estadual Paulista Júlio de Mesquita Filho

Fernanda Flavia Cockell Silva / Universidade Federal de São Paulo

Fernanda Sarreta / Universidade Estadual Paulista Júlio de Mesquita Filho

Filippina Chinelli / Fundação Oswaldo Cruz

Francini Vilela Novais / Universidade Federal de São Paulo 
Gaudêncio Frigotto / Universidade do Estado do Rio de Janeiro

Gerluce Alves Pontes da Silva / Ministério da Saúde

Gideon Borges dos Santos / Fundação Oswaldo Cruz

Giovana Zarpellon Mazo / Universidade do Estado de Santa Catarina

Gracia Maria de Miranda Gondim / Fundação Oswaldo Cruz

Greici Weinzierl / Universidade Federal de Santa Catarina

Ingrid D’avilla Freire Pereira / Fundação Oswaldo Cruz

Isabel Victoria Marazina / Universidade de São Paulo

Izabela Maria Rezende Taveira / Universidade Federal Fluminense

Jeane Freitas de Oliveira / Universidade Federal da Bahia

José Wellington Gomes Araujo / Fundação Oswaldo Cruz

Katia Morosov Alonso / Universidade Federal de Mato Grosso

Katia Virginia de Oliveira Feliciano / Instituto Materno Infantil de Pernambuco

Kenia Aparecida Miranda / Universidade Federal Fluminense

Laura Camargo Macruz Feuerwerker / Universidade de São Paulo

Leonardo Carnut / Universidade de Pernambuco

Leonir Lorenzetti / Universidade Alto Vale do Rio do Peixe

Lia Giraldo da Silva Augusto / Fundação Oswaldo Cruz

Lilian Miranda / Fundação Oswaldo Cruz

Liliana Muller Larocca / Universidade Federal do Paraná

Luciana Araújo dos Reis / Universidade Estadual do Sudoeste da Bahia 
Luciana de Almeida Colvero / Universidade de São Paulo

Luciane Maria Pezzato / Universidade Federal de São Paulo

Luciane Ouriques Ferreira / Fundação Oswaldo Cruz

Lucídio Bianchetti / Universidade Federal de Santa Catarina

Lucila Castanheira Nascimento / Universidade de São Paulo

Luiz Anildo Anacleto da Silva / Universidade Federal de Santa Catarina

Luiz Carlos Brant Carneiro / Universidade Federal de Minas Gerais

Magda Diniz Bezerra Dimenstein / Universidade Federal do Rio Grande do Norte

Magda Duarte dos Anjos Scherer / Universidade de Brasília

Marcelo Eduardo Pfeiffer Castellanos / Universidade Federal da Bahia

Marcelo Polacow Bisson / Faculdade de Medicina do ABC

Márcia Cavalcanti Raposo Lopes / Fundação Oswaldo Cruz

Márcia Schott / Universidade Estadual de Campinas

Margarita Antonia Villar Luis / Universidade de São Paulo

Maria Alice Pessanha de Carvalho / Fundação Oswaldo Cruz

Maria Amélia de Campos Oliveira / Universidade de São Paulo

Maria Ceci Araujo Misoczky / Universidade Federal do Rio Grande do Sul

Maria Constantina Caputo / Universidade Federal da Bahia

Maria das Graças Barbosa Moulin / Universidade Federal do Espírito Santo

Maria do Carmo Guedes / Pontifícia Universidade Católica de São Paulo

Maria Elizabeth Antunes Lima / Universidade Federal de Minas Gerais 
Maria Helena Mendonça / Fundação Oswaldo Cruz

Maria Luisa Sandoval Schmidt / Universidade de São Paulo

Maria Vilaní Cavalcante Guedes / Universidade Estadual do Ceará

Mariana Nogueira / Fundação Oswaldo Cruz

Marilia Bense Othero / Instituto Paliar

Marisa Lopes da Rocha / Universidade do Estado do Rio de Janeiro

Marise Nogueira Ramos / Fundação Oswaldo Cruz

Marisol de Paula Reis / Universidade Federal do Acre

Marluci Andrade Conceição Stipp / Universidade Federal do Rio de Janeiro

Marta Lenise do Prado / Universidade Federal de Santa Catarina

Maurício Monken / Fundação Oswaldo Cruz

Mauricio Vieira Martins / Universidade Federal Fluminense

Mônica de Castro Maia Senna / Universidade Federal Fluminense

Nádia Maciel Falcão / Universidade Federal do Amazonas

Patricia Lima Dubeux Abensur / Universidade Federal de São Paulo

Patricia Serpa de Souza Batista / Universidade Federal da Paraíba

Paulette Cavalcanti de Albuquerque / Fundação Oswaldo Cruz

Rafael Arouca Höfke Costa / Fundação Oswaldo Cruz

Raphael Mendonça Guimarães / Fundação Oswaldo Cruz

Regina Zanella Penteado / Universidade Metodista de Piracicaba

Rita de Cassia Padula Alves Vieira / Universidade Federal de Juiz de Fora 
Rodrigo Siqueira-Batista / Universidade Federal de Viçosa

Rosa Madeira Domingues / Fundação Oswaldo Cruz

Rosa Maria Castilhos / Universidade Federal do Rio Grande do Sul

Sandra Helena Isse Polaro / Universidade Federal do Pará

Sandra Lucia Correia Lima Fortes / Universidade do Estado do Rio de Janeiro

Silmara de Oliveira Gomes Papi / Universidade Estadual de Ponta Grossa

Silvana Nair Leite / Universidade do Vale do Itajaí

Silvia Angela Gugelmin / Universidade Federal de Mato Grosso

Silvia Beatriz Costa Czermainski / Secretaria de Estado da Saúde do Rio Grande do Sul

Silvia Regina Viodres Inoue / Universidade Federal de São Paulo

Simone Santos Oliveira / Fundação Oswaldo Cruz

Sonia Maria Dantas Berger / Universidade Federal Fluminense

Teresa Tonini / Universidade Federal do Rio de Janeiro

Thereza Maria Magalhães Moreira / Universidade Federal do Ceará

Umberto Catarino Pessoto / Secretaria de Estado da Saúde de São Paulo

Valéria Ferreira Romano / Universidade Federal do Rio de Janeiro

Vania Cardoso da Motta / Universidade Federal do Rio de Janeiro

Vera Lucia Navarro / Universidade de São Paulo

Viviane Laudelino Vieira / Universidade de São Paulo 